\title{
Kinetics of Temperature Response of PEO-b-PNIPAM-b-PAA Triblock Terpolymer Aggregates and of their Complexes with Lysozyme
}

Aristeidis Papagiannopoulos ${ }^{1, *}$, Anastasia Meristoudi ${ }^{1}$, Kunlun Hong $^{2}$ and Stergios Pispas ${ }^{1, *}$

${ }^{1}$ Theoretical and Physical Chemistry Institute, National Hellenic Research Foundation, 11635 Athens, Greece

${ }^{2}$ Center for Nanophase Materials Sciences, Oak Ridge National Laboratory, 2008 Oak Ridge TN, USA

\section{Corresponding Authors}

*E-mail: apapagiannopoulos@eie.gr. Tel.: +30210-7273825. Fax: +30210-7273794,

* E-mail: pispas@eie.gr. Tel.: +30210-7273824. Fax: +30210-7273794

\begin{abstract}
We present the kinetics of temperature response of a PEO-b-PNIPAM-b-PAA triblock terpolymer and of its complexes with lysozyme in aqueous solution. It is found that during the coil-to-globule transition of PNIPAM new bonds within the polymer aggregates are created, making the transition of the aggregates partially irreversible. This effect is also found for the protein loaded PEO-b-PNIPAM-b-PAA aggregates whereas in this case protein globules appear to enhance the formation of bonds, making the transition totally irreversible. The internal dynamics of both aggregates and complexes are "frozen" once the temperature is increased upon PINIPAM's LCST in water and remain so even when the temperature drops below LCST. We investigate the complexation kinetics of lysozyme and PEO-b-PNIPAM-bPAA and observe that it occurs in two stages, one where protein globules adsorb on single pre-formed aggregates and one where protein glubules cause inter-aggregate clustering.
\end{abstract}


Keywords: thermoresponsive polymers; protein complexation; kinetics

\section{INTRODUCTION}

Macromolecular compounds self-assembled in aqueous solutions, produce nano-scale formulations that are candidates for carriers in targeted drug and protein delivery, biosensors, and tissue engineering [1]. Nanoparticles with hydrophobic cores and hydrophilic coronas (neutral or polylectrolyte micelles) provide a template for drug loading either on the hydrophobic core via hydrophobic interactions [2] or via electrostatic attraction [3] with the corona (in the case of polyelectrolyte micelles). Charged or neutral nanogels [4] made of physically or chemically cross-linked macromolecules and polymeric aggregates [5] may also be utilized as pharmaceutical carriers. PNIPAM-containing macromolecules have been extensively studied and used in biomedical applications [6,7], because PNIPAM exhibits an LCST in aqueous solutions at about $32^{\circ} \mathrm{C}$ which is near the physiological temperature. PAA is a weak polyacid that is a common $\mathrm{pH}$-sensitive polyelectrolyte (with a $\mathrm{pK}_{\mathrm{a}}=4.2$ ) and $\mathrm{PEO}$ is a well-known water soluble, non-ionic and biocompatible polymer. Combination of these three blocks in one triblock terpolymer chain is expected to provide a rich content of stimuli responsive properties to a water soluble biocompatible polymeric compound.

The scope of this work is to investigate the properties of a macromolecular system that has both protein-loading properties and the inherent ability to respond to thermal stimuli. Similar systems [8,9] have been studied in terms of these capabilities. In the triblock terpolymer under study temperature increase induces additional clustering in the preformed aggregates and in addition the complexed proteins influence this response. We use Multi-Angle Laser Light Scattering (M.A.L.L.S.) to study the kinetics of: 1) the temperature response of PEO-bPNIPAM-b-PAA with temperature jumps from below to above the LCST of PNIPAM and 
vice-versa, 2) the complexation between PEO-b-PNIPAM-b-PAA with the model globular protein lysozyme and 3) the temperature response of the formed triblock terpolymer/protein complexes. The feature of multi-detection at many angles simultaneously allows us to shed light to the morphological transitions of a complexed polyelectrolyte/protein system whith inherent thermoresponsive nature.

\section{EXPERIMENTAL SECTION}

The tri-block terpolymer poly(ethylene oxide)-block-poly( $\mathrm{N}$-isopropylacrylamide)-blockpoly(acrylic acid) (PEO-b-PNIPAM-b-PAA) was synthesized using reversible additionfragmentation chain transfer (RAFT) polymerization [10,11]. The resulting $\mathrm{M}_{\mathrm{w}}$ is $\mathrm{M}=56 \cdot 10^{3}$ g/mol with respective weight contents: $7 \%$ for PEO, $68 \%$ for PNIPAM and $25 \%$ for PAA (giving the following number-average degrees of polymerization: PEO$_{89}-\mathrm{PNIPAM}_{337}-$ PAA 194 ) and a polydispersity index $\mathrm{M}_{\mathrm{w}} / \mathrm{M}_{\mathrm{n}}=1.28$ from SEC in DMF (see Supplementary Material). The polymer was dissolved in water $(\mathrm{pH} 7)$ at $0.1 \mathrm{mg} / \mathrm{ml}$. The samples were left overnight to equilibrate and the salt content was set afterwards to $0.01 \mathrm{M} \mathrm{NaCl}$. Lysozyme (HEWL) with molecular weight $\mathrm{M}=14.7 \cdot 10^{3} \mathrm{~g} / \mathrm{mol}$ was purchased from Fluka and used without further purification. Stock solutions were prepared by dissolving lysozyme at 0.1 $\mathrm{mg} / \mathrm{ml}$ in the $\mathrm{pH} 7 / 0.01 \mathrm{M} \mathrm{NaCl}$ aqueous medium and leaving overnight to equilibrate. All samples were filtered by $0.45 \mu \mathrm{m}$ hydrophilic sartorious filters in order to eliminate dust or large clusters and loaded into standard 1cm width Helma quartz dust - free cells.

Multi Angle Laser Light Scattering was performed with an ALV compact goniometer system (ALVGmbH, Germany), equipped with four ALV-5000/EPP multi tau digital correlators and a He-Ne laser operating at the wavelength of $632.8 \mathrm{~nm}$ able to perform a full static and dynamic measurement at 16 angles (from 20 to $146^{\circ}$ ) in 7 minutes. In Static Light Scattering (SLS) the Rayleigh ratio [12] $R(q)$ is written as:

$\frac{K c}{R(q, c)}=\frac{1}{M_{w} P(q)}$ 
$M_{w}$ is the weight-averaged molar mass and $c$ is the particle mass-concentration in solution. The single particle's form factor is given $P(q)=e^{-\frac{1}{3} q^{2} R_{g}^{2}}$ (Guinier approximation), where $R_{g}$ the (z-averaged) radius of gyration.

In Dynamic Light Scattering [12] (DLS) the intensity auto-correlation functions $g^{(2)}(t)$ at different scattering angles analysed by the CONTIN algorithm. The characteristic relaxation rate $\Gamma(q)$ is taken from the position of the maximum $(\tau(q))$ of the distribution function of relaxation times $\left(\Gamma(q)=\frac{1}{\tau(q)}\right)$ and in the case of diffusive modes the diffusion coefficient $D$ is obtained $\left(\Gamma(q)=D \cdot q^{2}\right)$. The hydrodynamic radius $\left(R_{h}\right)$ is extracted from the StokesEinstein equation (equation 2).

$R_{h}=\frac{k_{B} T}{6 \pi \eta D}$

where $\eta$ is the viscosity of the solvent, $k_{B}$ is the Boltzmann constant and $T$ is the absolute temperature. The temperature of the samples was set by a PolyScience temperature controller. For an increasing-temperature jump the sample cell was removed from the sample holder, the sample holder/toluene bath was heated to the desired temperature and the sample was rapidly inserted into the sample environment. For the decreasing-temperature jumps the desired temperature was set in the bath without removing the sample and going on with the data collection. The temperature inside the sample-containing toluene bath did not take longer than 5 minutes to reach the set point.

\section{RESULTS AND DISCUSSION}

\subsection{Kinetics of PEO-b-PNIPAM-b-PAA assembly}

In Figure 1a the SLS data from a 30 to $35{ }^{\circ} \mathrm{C}$ temperature jump (see Supplementary Material for critical temperature of the aqueous solutions of this work) is shown for several instants after the temperature jump in Guinier representation. The low- $q$ range of our LS measurements allows determination of $R_{g}$ values up to $\sim 200-250 \mathrm{~nm}$ when the first 3-4 first 
data points are taken into account (see Supplementary Material about Guinier analysis). As it can be shown [13] the Guinier approximation estimates $R_{g}$ with $10 \%$ accuracy up to $q R_{g} \approx$ 2. The divergence from linearity at higher $q$ can be attributed to effects of the form factor of the scattering objects [14] or co-presence of species of smaller $R_{g}$. In DLS (Figure 1b) the linear initial part of the $\Gamma(q)$ vs $q^{2}$ plots was used to extract $R_{h}$. The divergence of these plots from linearity at higher $q$ can be attributed to either internal dynamics [15] or copresence of species with higher diffusion coefficient. The analysis of the data is similar for the rest of the study in terms of measurement of $R_{g}, R_{h}$ and $M_{w}$.
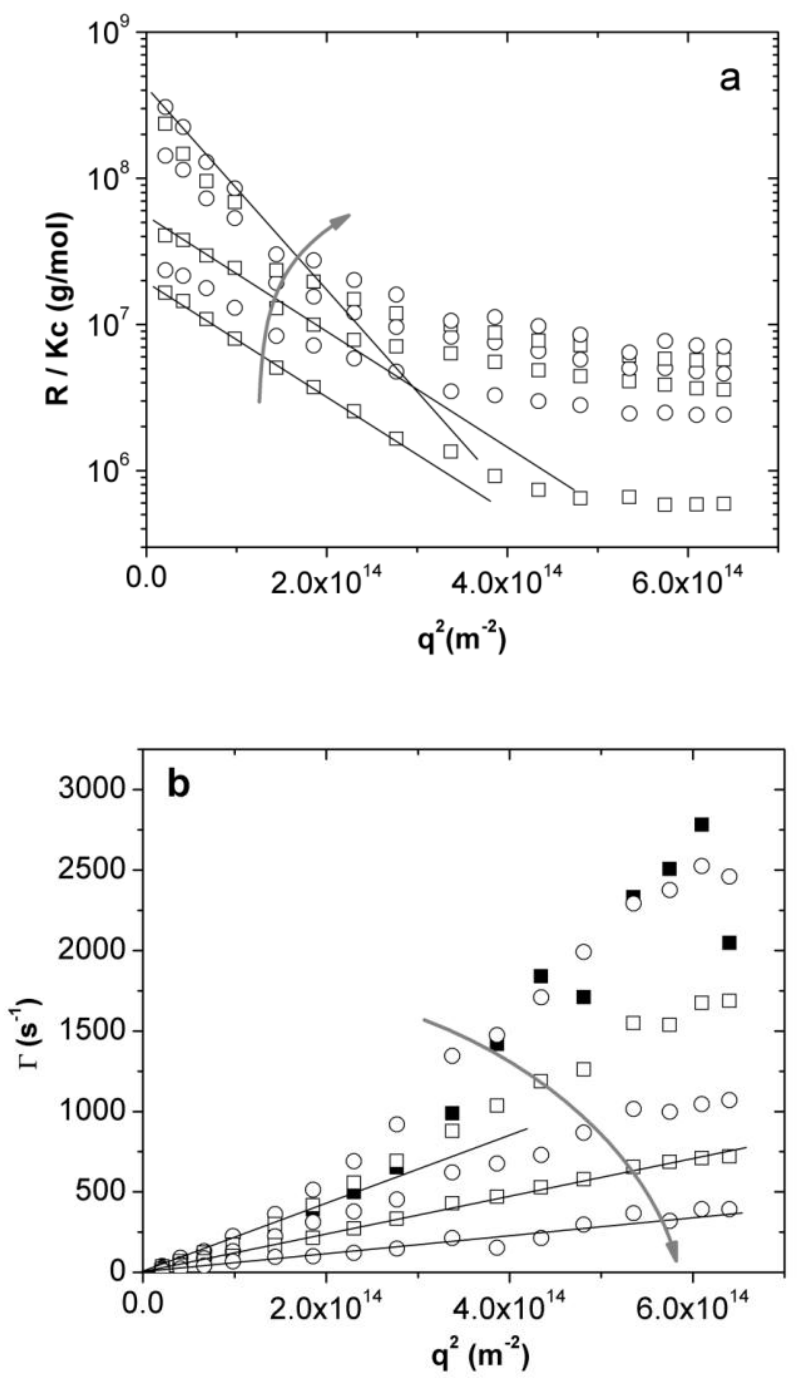
Figure 1. SLS (a) and DLS (b) data from a 30 to $35{ }^{\circ} \mathrm{C}$ temperature jump for PEO-bPNIPAM-b-PAA $(0.1 \mathrm{mg} / \mathrm{ml}, \mathrm{pH} 7$ and $0.01 \mathrm{M} \mathrm{NaCl})$ from measurements about $25 \mathrm{~min}$ apart from each other. Arrows represent the passing of time. Straight lines are fits at the initial linear part of the data sets. Filled squares represent the data at the start of the experiment (hence they do not follow the time-arrow).

The apparent aggregation number $N_{a g g}$ (number of triblock chains per aggregate) is found much higher than unity already from $30{ }^{\circ} \mathrm{C}$ (the data was unchanged between 25 to $30{ }^{\circ} \mathrm{C}$ ). In aqueous solutions the expected unperturbed dimensions of PEO [16] $\left(R_{g} \approx 3 n m\right)$, PNIPAM [17] $\left(R_{g} \approx 8 \mathrm{~nm}\right.$, below LCST) and PAA [18] ( $\mathrm{F} \approx 50 \mathrm{~nm}$, fully stretched $)$ chains of similar molecular weights are small compared to the apparent sizes observed by LS. Hence, the system is in an aggregated state possibly due to hydrophobic attraction between PNIPAM blocks below LCST [19,20]. Also hydrogen bonding between PEO and PAA [12] blocks is a possible cause of inter-chain associations. Although hydrogen bonding is strong at low $\mathrm{pH}$, it may stabilize aggregated structures in a cooperative manner in neutral or even weak basic conditions. This has been shown in [21] where the PAA-g-PEO copolymer showed aggregation at high $\mathrm{pH}$ contrary to the mixture of linear homopolymer PAA and PEO. There, it was claimed that the entropic cost for aggregation is lower in the case of preajoined PEO segments in a PAA backbone. In the first 25 minutes after the increase-temperature jump there is a mild increase in $N_{a g g}$ and a simultaneous decrease in the radii (Figure 2) caused by the coil-to-globule transition of PNIPAM block [22]. The mild increase of $N_{a g g}$ signifies the enhanced presence of hydrophobic units in the aggregates that facilitates further aggregation although at this stage this is not the dominant effect. In the recent works of Papadakis et al. the thermoresponsive kinetic studies of PS-b-PNIPAM-b-PS flower-like micelles reveal an 
initial stage of intermicellar morphological rearrangement (collapse of the PNIPAM shell) and a subsequent intermicellar clustering [23,24].

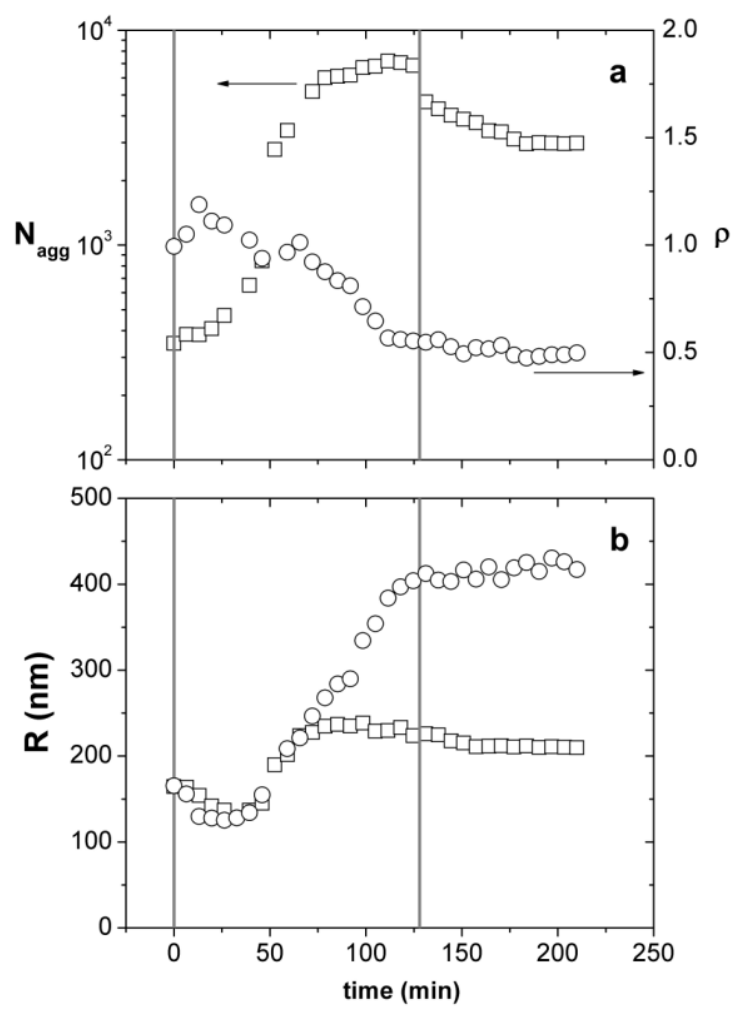

Figure 2. $N_{a g g}$ (squares) and $\rho=\frac{R_{g}}{R_{h}}$ (circles) (a) and $R_{g}$ (squares) and $R_{h}$ (circles) (b) for PEO-b-PNIPAM-b-PAA $(0.1 \mathrm{mg} / \mathrm{ml}, \mathrm{pH} 7$ and $0.01 \mathrm{M} \mathrm{NaCl})$. Vertical lines indicate the jump from 30 to $35{ }^{\circ} \mathrm{C}$ (left) and from 35 to $30{ }^{\circ} \mathrm{C}$ (right).

During the initial stage ( 0 to $25 \mathrm{~min}$ ) the ratio $\rho=\frac{R_{g}}{R_{h}}$ increases from $\rho \approx 1$ to $\rho \approx 1.2$. This is compatible with a morphology of a randomly cross-linked microgel [25] or a dense fractal aggregate [26]. Between 50 and 100 minutes there is a strong increase in $N_{a g g}$ due to strong clustering between aggregates.

The increase in $N_{a g g}$ is accompanied by a strong increase in $R_{h}$. On the other hand $R_{g}$ reaches a plateau value at 75 minutes and consequently from that time $\rho$ drops below 1 
eventualy reaching a value between 0.5 and 0.6 , reminescent of a dense core-diffuse shell morphology [27]. We had previously observed a super-core-shell spherical morphology [20] of flower-like PS-b-PNIPAM-b-PS micelles within aggregates caused by the presence of PNIPAM. Since after 75 minutes $R_{g}$ is constant while $R_{h}$ increases we conclude that at this time-regime aggregates adhere loosely on pre-formed denser aggregates and at the same time the initial core of the clusters collapses further. Values of $\rho$ below the one of a uniform sphere [28] ( 0.775) can be observed also for microgels[29] and there also exist observations where a dense core can further collapse while the outer periphery remains virtually unchanged, leading to a decrease in $R_{g}$ while $R_{h}$ remains constant [25].

In PEO-b-PNIPAM aqueous solutions [30,19] temperature induced aggregation has led to formation of vesicles. In our PAA containing copolymers there is no sign of this kind of morphology. Interestingly vesicles were found in another PAA containing system [31] but at that case the molecular weight of the PAA block $\left(\sim 10^{3} \mathrm{~g} / \mathrm{mol}\right)$ was much lower than ours $\left(14 \cdot 10^{3} \mathrm{~g} / \mathrm{mol}\right)$ and was the middle block of the terpolymer (PEO-b-PAA-b-PNIPAM). The same group used their procedure of forming the terpolymer polymersomes at $40{ }^{\circ} \mathrm{C}$ to simultaneously incorporate proteins of various kinds [8].

Switching the temperature from 35 back to $30^{\circ} \mathrm{C}$ (Figure 2) there is a weak drop in $N_{\text {agg }}$ which reaches a plateau value much higher than the one before the temperature-increase jump. Apparently the PAA-PEO and PAA-PAA contacts that are formed at $35^{\circ} \mathrm{C}$ when PAA and PEO groups are forced to come closer within the clusters, persist after the swelling of PNIPAM. PAA is an intrinsically hydrophobic polyelectrolyte. Additionally, PAA may form collapsed layers, as in the case of core-shell polyelectrolyte micelles with a hydrophobic core $[18]$.

In many studies of aqueous solutions of PNIPAM-b-PEO diblock copolymers the temperature induced transitions have been reported to be totally reversible [30]. In a 
spectroscopy study of PNIPAM-co-AA hydrogels the irreversibility of the transition was attributed to the formation of internal hydrogen bonds between acrylic acid groups during the volume phase transition. Additionally in another thermoresponsive system [32] the close proximity of monomers caused by the thermoresponsive transition led to formation of $\mathrm{COOH}-\mathrm{COO}^{-}$hydrogen bonds [33].

There is a very weak increase in $R_{h}$ (at the jump from 35 back to $30^{\circ} \mathrm{C}$ ). The increase in $R_{h}$ and dissociation (to some extent) of the clusters (decrease in $N_{a g g}$ ) show a relaxed conformation of the clusters below the LCST. This small increase in $R_{h}$ is most probably due to the extension of PNIPAM blocks and the resulting swelling and hydration of the clusters [34].

\subsection{Kinetics of complexation and temperature response of PEO-b-PNIPAM-b-PAA /}

\section{lysozyme complexes}

Mixing the two oppositely charged components (time=0) - PEO-b-PNIPAM-b-PAA and lysozyme - at room temperature results in complexation (Figure 3). For the complexed amount of lysozyme per PEO-b-PNIPAM-b-PAA aggregate the procedure of Kokufuta et al. is used [35] (see Supplementary Material). For about 80 minutes (right vertical line of Figure 3) the radii increase weakly (Figure $3 \mathrm{a}$ ) while their ratio is fairly constant. $N_{\text {glob }}$ increases strongly until about 50 minutes and reaches a plateau between 50 and 80 minutes. The weak increase in radii and the stability of their ratio shows that the aggregates do not change their morphology significantly during complexation, while lysozyme globules cause a mild swelling upon the aggregates. 


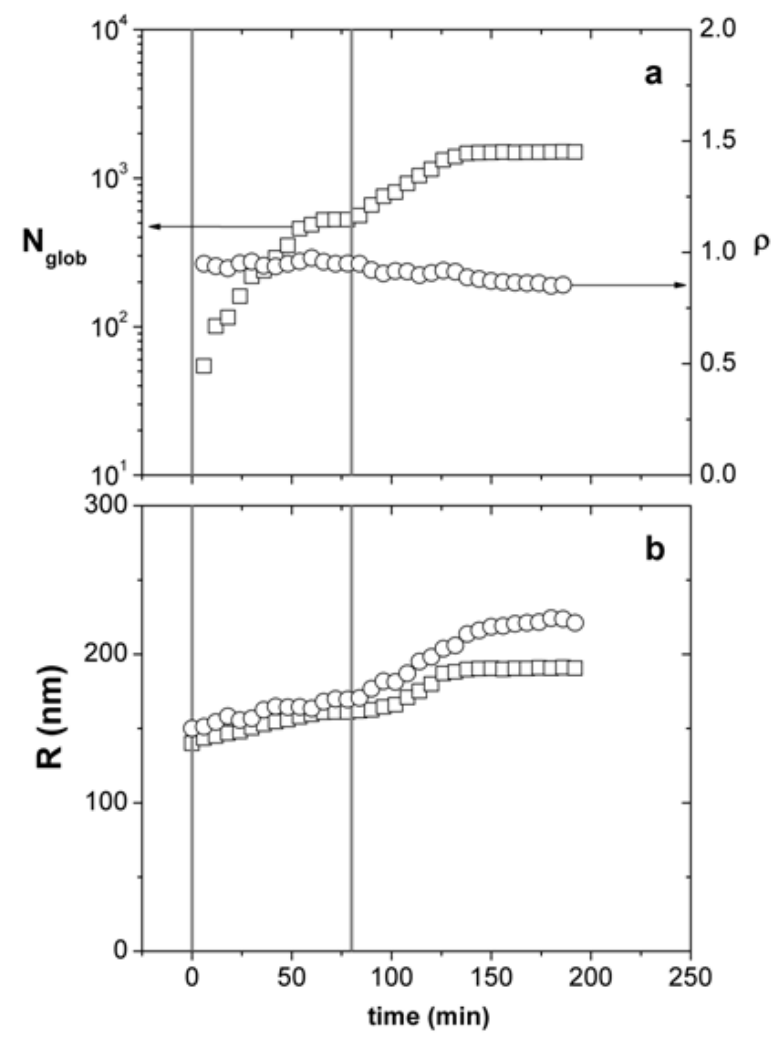

Figure 3. Number of lysozyme globules per micelle (squares) and $\rho$ (circles) (a) and $R_{g}$ (squares) and $R_{h}$ (circles) (b) for PEO-b-PNIPAM-b-PAA $(0.1 \mathrm{mg} / \mathrm{ml}, \mathrm{pH} 7$ and $0.01 \mathrm{M}$ $\mathrm{NaCl})$ during complexation with lysozyme $(0.025 \mathrm{mg} / \mathrm{ml}, \mathrm{pH} 7$ and $0.01 \mathrm{M} \mathrm{NaCl})$. The experiment is run at $25^{\circ} \mathrm{C}$.

After 80 minutes to the end of the experiment $N_{g l o b}$ and radii increase (up to $\sim 150$ minutes) and reach a plateau. The final value of $\rho \approx 0.85$ indicates a trend of the clusters to form a more compact spherical structure. At 80 minutes the total amount of complexed lysozyme is approximately equal to the amount of lysozyme dissolved in solution. This means that there is no free lysozyme left in solution. In Dubin et al. [36] it is shown that BSA/PDADMAC coacervation may lead up to $100 \%$ separation of BSA from solution, when the molecular weight of PDADMAC exceeds $100 \mathrm{k} \mathrm{g} / \mathrm{mol}$. Another example is the work of Cousin et al. [37] where the formation of lysozyme/PSS complexes are studied. It is found that when the charge ratio of introduced PSS and lysozyme in solution is near 1 then there is no free 
lysozyme in solution. Hence, values $N_{\text {glob }}>500$ are apparently caused by clustering between aggregates.

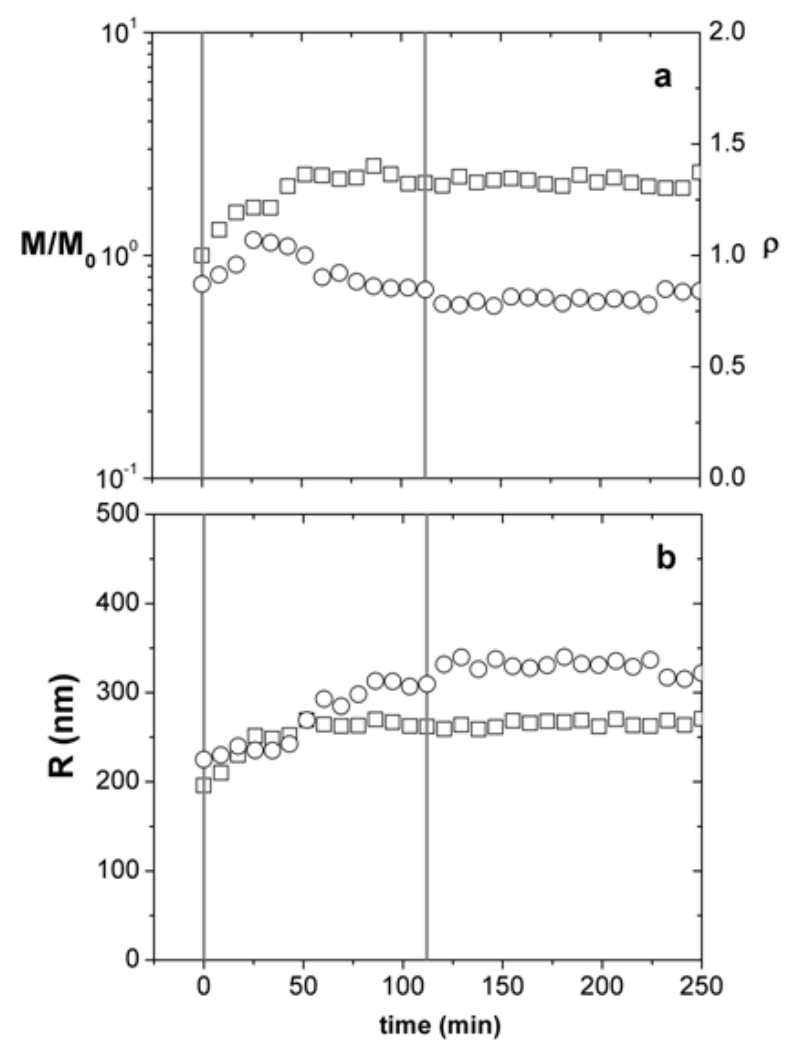

Figure 4. Relative molecular weight (squares) and characteristic ratio (circles) (a) and $R_{g}$ (squares) and $R_{h}$ (circles) (b) for PEO-b-PNIPAM-b-PAA/lysozyme complexes $(0.1 / 0.025 \mathrm{mg} / \mathrm{ml}, \mathrm{pH} 7$ and $0.01 \mathrm{M} \mathrm{NaCl})$. The vertical lines indicate the jump from 30 to 35 ${ }^{\circ} \mathrm{C}$ (left) and from 35 to $30{ }^{\circ} \mathrm{C}$ (right).

In Figure 4 the kinetics of the formed PEO-b-PNIPAM-b-PAA / lysozyme complexes through the two temperature jumps is shown. Upon the increase in temperature the molecular weight of the clusters increases for 70-80 minutes before it reaches equilibrium. $M / M_{0}$ is the ratio of the apparent molecular weight at a certain time over the molecular weight of the clusters at the first temperature jump $(\mathrm{t}=0)$. The increased hydrophobicity of the PNIPAM blocks cause further clustering of the complexes increasing the molecular weight by 2-3 
times while the radii increase mildly $\left(R_{g}\right.$ reaches a plateau sooner than $\left.R_{h}\right)$ and $\rho$ decreases towards a compact spherical conformation. Droping the temperature to $30{ }^{\circ} \mathrm{C}$ the complexes remain virtually unaltered. Probably the bonds created while PNIPAM is in its globule state and caused the partial irreversibility in the case of the pure polymer aggregates (with no added lysozyme) are now enhanced by the presence of lysozyme which also contains hydrophobic domains. This way the combination of lysozyme and PEO-b-PNIPAM-b-PAA aggregates create a hybrid nanostructure that is irreversibly transformed to a compact structure upon the increasing-temperature jump.

At this point it is worth commenting on the details of the $\Gamma(q)$ vs $q^{2}$ of this study (see Supplementary Material). The plots are initially highly non-linear and eventually linear after some time well within the increasing-temperature jump. Even after the decrease in temperature back to $30{ }^{\circ} \mathrm{C}$ the plots remain linear for both PEO-b-PNIPAM-b-PAA aggregates and PEO-b-PNIPAM-b-PAA/lysozyme complexes. For all the experiments the distributions of relaxation rates is fairly narrow and apparently dominated by a single species (see Supplementary Material). In DLS the correlated fluctuations at low-q $\left(q R_{g} \ll 1\right)$ are governed by the brownian diffusion of the scattering particles. At high-q $\left(q R_{g} \gg 1\right)$ the internal particle fluctuations are observed. From our SLS data it is clear that the separation of the q-regimes falls within the experimental q-window. Hence the non-linearity of the $\Gamma(q)$ vs $q^{2}$ plots is due to internal dynamic relaxations of the aggregates [38]. Upon increase of temperature the stronger binding between chains (and/or chain-lysozyme in the case of complexes) restricts the internal dynamics and the plots become linear (internally rigid bodies). This "freezing" remains after the return of temperature below LCST, which is in accordance to the partial irreversibility of the aggregates and the total irreversibility of the complexes. In the case of complexation of PEO-b-PNIPAM-b-PAA with lysozyme below 
LCST the non-linearity of the plots is observed through the whole experiment showing that lysozyme allows internal dynamical relaxations during its binding.

\section{CONCLUSIONS}

M.A.L.L.S. was used to characterize thermoresponsiveness and complexation in PEO-bPNIPAM-b-PAA / lysozyme aqueous solutions. At room temperature PEO-b-PNIPAM-bPAA form aggregates that upon increase in temperature from 30 to $35^{\circ} \mathrm{C}$ initially shrink and finally create inter-aggregate clusters. The initial microgel/fractal aggregate structure changes to an apparently core-shell-like morphology. The kinetics of complexation of lysozyme with the aggregates (room temperature) is initially dominated by the loading of protein globules on aggregates and finally by protein-mediated inter-aggregate clustering. This clustering is further enhanced upon increase of temperature. Interestingly, there is evidence that internal dynamics of PEO-b-PNIPAM-b-PAA aggregates and PEO-b-PNIPAM-b-PAA / lysozyme complexes "freeze" irreversibly during the increasing-temperature-jump. This study proves the importance of the kinetics studies in these systems and creates ground for further analytical experiments as a function of polymer concentration, target temperatures, salt content and $\mathrm{pH}$ in order to reveal the responsiveness of the self-assembled nanosystems. Finally the irreversibility properties of the system shows the possibility of taylormaking nanoparticles with thermal treatment.

\section{ACKNOWLEDGEMENTS}

Authors acknowledge financial support of the work by the NANOMACRO 1129 project which is implemented in the framework of the Operational Program "Education and Lifelong Learning" (Action "ARISTEIA I") and it is co-funded by the European Union (European Social Fund) and by national funds. Light scattering was conducted at the Center for Nanophase Materials Sciences, which is a DOE Office of Science User Facility. 


\section{REFERENCES:}

[1] Stuart, M. A. C.; Huck, W. T. S.; Genzer, J.; Muller, M.; Ober, C.; Stamm, M.;

Sukhorukov, G. B.; Szleifer, I.; Tsukruk, V. V.; Urban, M.; Winnik, F.; Zauscher, S.;

Luzinov, I.; Minko, S. Nat Mater 2010, 9, (2), 101-113.

[2] Torchilin, V. P. Journal of Controlled Release 2001, 73, (2-3), 137-172.

[3] Ballauff, M. Prog. Polym. Sci. 2007, 32, 1135-1151.

[4] Kabanov, A.; Vinogradov, S., In Multifunctional Pharmaceutical Nanocarriers, Torchilin, V., Ed. Springer New York: 2008; Vol. 4, pp 67-80.

[5] Ryu, J.-H.; Chacko, R. T.; Jiwpanich, S.; Bickerton, S.; Babu, R. P.; Thayumanavan, S. Journal of the American Chemical Society 2010, 132, (48), 17227-17235.

[6] Klouda, L.; Mikos, A. G. European Journal of Pharmaceutics and Biopharmaceutics 2008, 68, (1), 34-45.

[7] Hoare, T. R.; Kohane, D. S. Polymer 2008, 49, (8), 1993-2007.

[8] Welsch N.; Ballauff, M.; Lu, Y. Adv. Polym. Sci. 2010, 234, 129-163.

[9] Cheng, R.; Meng, F.; Ma, S.; Xu, H.; Liu, H.; Jing, X.; Zhong, Z. Journal of Materials Chemistry 2011, 21, 19013-19020.

[10] Zhao, J.; Zhang, G.; Pispas, S. Journal of Polymer Science Part A: Polymer Chemistry 2009, 47, (16), 4099-4110.

[11] Moad, G.; Rizzardo, E.; Thang, S. H. Aust. J. Chem. 2009, 62, (11), 1402-1472.

[12] Papagiannopoulos A., Karayianni M., Mountrichas G., Pispas S, Radulescu A., Polymer 2015, 63, 134-143.

[13] Feigin, L. A.; Svergun, D. I., Structure Analysis by Small-Angle X-Ray and Neutron Scattering. Plenum Press, New York and London, 1987.

[14] Sorensen, C. M. Aerosol Sci. Technol. 2001, 35, (2), 648-687.

[15] Chu, B.; Wang, Z.; Yu, J. Macromolecules 1991, 24, (26), 6832-6838. 
[16] Devanand, K.; Selser, J. C. Macromolecules 1991, 24, (22), 5943-5947.

[17] Kubota, K.; Hamano, K.; Kuwahara, N.; Fujishige, S.; Ando, I. Polym J 1990, 22, (12), 1051-1057.

[18] Colombani, O.; Ruppel, M.; Burkhardt, M.; Drechsler, M.; Schumacher, M.;

Gradzielski, M.; Schweins, R.; Müller, A. H. E. Macromolecules 2007, 40, (12), 43514362.

[19] Papagiannopoulos, A.; Zhao, J.; Zhang, G.; Pispas, S.; Radulescu, A. Polymer 2013, 54, (23), 6373-6380.

[20] Papagiannopoulos, A.; Zhao, J.; Zhang, G.; Pispas, S.; Radulescu, A. European Polymer Journal 2014, 56, (0), 59-68.

[21] Hao, J.; Yuan, G.; He, W.; Cheng, H.; Han, C. C.; Wu, C. Macromolecules 2010, 43, 2002-2008.

[22] Wu, C.; Wang, X. Physical Review Letters 1998, 80, (18), 4092-4094.

[23] Adelsberger, J.; Grillo, I.; Kulkarni, A.; Sharp, M.; Bivigou-Koumba, A. M.; Laschewsky, A.; Muller-Buschbaum, P.; Papadakis, C. M. Soft Matter 2013, 9, (5), $1685-1699$.

[24] Adelsberger, J.; Metwalli, E.; Diethert, A.; Grillo, I.; Bivigou-Koumba, A. M.; Laschewsky, A.; Müller-Buschbaum, P.; Papadakis, C. M. Macromolecular Rapid Communications 2012, 33, (3), 254-259.

[25] Schmitz, K. S.; Wang, B.; Kokufuta, E. Macromolecules 2001, 34, (23), 8370-8377.

[26] Van Saarloos, W. Physica A 1987, 147, 280-296.

[27] Hirzinger, B.; Helmstedt, M.; Stejskal, J. Polymer 2000, 41, (8), 2883-2891.

[28] Müller, A.; Burchard, W. Colloid Polym Sci 1995, 273, (9), 866-875.

[29] Boyko, V.; Pich, A.; Lu, Y.; Richter, S.; Arndt, K.-F.; Adler, H.-J. P. Polymer 2003, 44, (26), 7821-7827. 
[30] Qin S.; Geng Y.; Discher D. E.; Yang S. Advanced Materials 2006, 18, 2905-2909.

[31] Xu, H.; Meng, F.; Zhong, Z. Journal of Materials Chemistry 2009, 19, 4183-4190.

[32] Sun, S.; Hu, J.; Tanga, H.; Wu, P. Physical Chemistry Chemical Physics 2011, 13, $5061-5067$.

[33] Sedlak, M. The Journal of Physical Chemistry B 2012, 116, 2356-2364

[34] Hirokawa, Y.; Tanaka, T. The Journal of Chemical Physics 1984, 81, (12), 6379-6380.

[35] Azegami, S.; Tsuboi, A.; Izumi, T.; Hirata, M.; Dubin, P. L.; Wang, B.; Kokufuta, E. Langmuir 1999, 15, (4), 940-947.

[36] Wang, Y.; Gao, J. Y.; Dubin, P. L. Biotechnology Progress 1996, 12, 356-362

[37] Gummel, J.; Boue, F.; Deme, B.; Cousin, F. The Journal of Physical Chemistry B 2006, $110,24837-24846$.

[38] Galinsky, G.; Burchard, W. Macromolecules 1997, 30, (22), 6966-6973. 